\title{
Solvent neurotoxicity in vehicle collision repair workers in New Zealand.
}

Samuel Keer ${ }^{1}$, Bill Glass ${ }^{1}$, Bradley Prezant ${ }^{1}$, David McLean ${ }^{1}$, Neil Pearce ${ }^{2}$, Elizabeth Harding ${ }^{1}$, Diana Echeverria ${ }^{3}$, James McGlothlin ${ }^{4}$, Duncan Babbage ${ }^{5}$, Jeroen Douwes ${ }^{1}$.

${ }^{1}$ Centre for Public Health Research, Massey University, Wellington, New Zealand

${ }^{2}$ Department of Medical Statistics, London School of Hygiene and Tropical Medicine, London, United Kingdom ${ }^{3}$ School of Public Health, Washington University, Washington, U.S.A.

${ }^{4}$ School of Health Sciences, Purdue University, West Lafayette, U.S.A.

${ }^{5}$ Centre for eHealth, Auckland University of Technology, Auckland, New Zealand

Correspondence:

Samuel Keer, Centre for Public Health Research, Massey University.

Private Box 756, Wellington, New Zealand

E-Mail-s.keer@massey.ac.nz

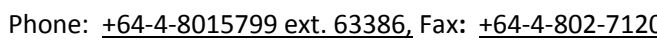

Word Count: $\mathbf{3 4 0 3}$

Keywords: Solvents, neurobehavioural, occupational, spray painters, panel beaters 


\section{ABSTRACT}

Objectives: To assess whether ehanges in paint formulations, solvent use and workplace practices introduced in the vehicle collision repair industry arer over the past few decades have reduced solvent exposures and associated with neurotoxicity in spray painters and panel beaters.

Methods: Neurobehavioural symptoms were assessed in 370 vehicle collision repair and 215 reference workers using the EUROQUEST questionnaire. Full-shift airborne solvent levels were measured in a subset $(n=92)$ of collision repair workers.

Results: Solvent exposures were higher in spray painters than in panel beaters, but levels were below current international exposure standards. Collision repair workers were more likely to report symptoms of neurotoxicity than reference workers with ORs of $2.0,2.4$ and 6.4 (all $p<0.05$ ) for reporting $\geq 5,>10$ and $\geq 15$ symptoms respectively. Panel beaters generally had the greatest number of symptoms. Associations with specific symptom domains showed increased risks for neurological (OR 4.2), psychosomatic (OR 3.2), mood (OR 2.1), memory (OR 2.9) and memory and concentration symptoms combined (OR 2.4) (all $p<0.05)$. Workers who had worked for 10-19 years (average 15) or 20+ years (an-average of 15-and-31) 1 years in the collision repair industry reported consistently more symptoms than those who had only worked less than 10 years (en average 5)-years, even after adjusting for age. However, those who worked more than 20 en average 31 years generally reported fewer symptoms than those who worked 10 -195 years, suggesting a possible healthy worker effect.

Conclusions: Despite low airborne solvent exposures, vehicle collision repair spray painters and panel beaters continue to be at risk of symptoms of neurotoxicity. 


\section{INTRODUCTION}

Acute health effects of occupational exposure to solvents (e.g. headaches, nausea and light-headedness ) have long been recognised, with very high exposures associated with intoxication, unconsciousness and in some cases death [1]. Chronic effects such as sustained changes in mood, memory, concentration and cognitive deficits have also been documented, in some cases leading to a diagnosis of Chronic Solvent Neurotoxicity (CSN) or Chronic Toxic Encephalopathy (CTE) [1-4]. Among those with CSN, industrial and automotive repair spray painters are consistently over-represented [5]. Spray painting involves the use of large quantities of solvent mixes for preparation work, cleaning of refurbished panels, and thinning of paint. This and the subsequent spraying of solvent-containing paints may result in elevated solvent exposure through both inhalation and dermal absorption [1].

Several cross-sectional studies since the 1970's have shown memory and concentration deficits in spray painters and other solvent-exposed workers [6-10], but findings have not always been consistent [11-13]. Dose-response associations have been reported [14] but are often weak, possibly due to the 'healthy worker effect' [15]. Also, previous studies have often been conducted intargeted larger enterprises where workplace hygiene and hazards are likely to be managed more effectively [9 16] and studies were not always adequately controlled for potential confounders [15 17 18].

Significant changes in paint formulations, solvent use and workplace practices have occurred in this industry in the past few decades which, as suggested recently [19], may have resulted in a significant decline in workplace solvent exposures. However, little research has been conducted to confirm this and it is also unclear whether this has contributed to a significantly reduced risk of neurotoxicity in collision repair workers.

In the current study, collision repair industry workers $(n=370)$ from small to medium workshops, and a reference group of construction industry workers $(n=215)$ in New Zealand were recruited to assess contemporary solvent exposures and associated neurotoxicity risks. 


\section{METHODS}

\section{Study Population}

The study population was recruited from collision repair workshops throughout the North Island of New

Zealand, with a focus on the main centres (Wellington and Auckland). Workshops were identified from the Yellow Pages and internet searches and 175 workshops each employing between 2 and 15 staff were recruited. All staff aged between $17-70$ years were invited to take part, including spray painters, panel beaters (or auto body repair workers) and office staff with a history of work as a spray painter or panel beater. Exclusion criteria were any history of major head injury or neurological/neurodegenerative disease, including meningitis, major depression or epilepsy. Collision repair workers who declined participation were invited to complete a short questionnaire assessing key demographic factors, symptoms of neurotoxicity and lifestyle factors. A small group of office workers with no history of exposure-work were also invited to take part in exposure sampling. A reference group of construction workers with negligible/no exposure to solvents was recruited in the same regions using a similar strategy and exclusion criteria.

Commented [n3]: This is a bit confusing; how many were approached? Did 175 agree or was that the number approached?

\section{Questionnaire}

Information on demographics, work characteristics, use of solvents and solvent-based products and other potential confounders was obtained for all participants by questionnaire. Current (i.e. in the past 3 months) symptoms of neurotoxicity were measured using an adapted version of the EUROQUEST [20] questionnaire, administered face-to-face. The questionnaire consists of 59 core items, which cover the following symptom domains: neurological (e.g. numbness and tingling in extremities, balance problems etc.), psychosomatic (headaches, nausea, tinnitus etc.), mood, memory, concentration, fatigue and sleep quality. EUROQUEST also includes questions on symptoms of acute exposure (irritation of the mucosal membranes and intoxication, 6 items). Symptom frequency for these and the 59 core symptoms in recent months was reported on a 4-point scale, "Seldom or Never", "Sometimes", "Often" or "Very often". Questions regarding sensitivity to environmental conditions (6 items, e.g. "Are you sensitive to bright lights?") and anxiety (6 items, e.g. "Are you generally a nervous person?") were also included and rated on a different 4-point scale ("Strongly disagree", "Disagree", "Agree" or "Strongly agree"). The final section of the EUROQUEST assesses perceived general 
health (4 items), where participants are asked to rate different aspects of their general health and wellness as "Very good" "Good", "poor" or "Very poor". For the purpose of subsequent analyses we dichotomised symptoms, with "Strongly disagree" or "Disagree", "Seldom or Never" or "sometimes", and "poor" or "very poor" constituting a negative response and "Agree" or "Strongly agree", "Often" or "Very often", "very good" and "good" constituting a positive response[21]. Anxiety and perceived general health were included to enable us to control the analyses for individual personality traits which have been found to lead participants to under or over report their symptoms [21].

\section{Exposure assessment}

Full-shift airborne personal exposure measurements were conducted in 17 collision repair workshops using a whole-air method [22]; these, and included spray painters $(n=50)$, panel beaters $(n=36)$, and a group of office workers $(n=6)$. Teflon tubing running from the workers breathing zone was connected to a $400 \mathrm{cc}$ stainless steel sampling canister (Restek Corporation, PA, USA) negatively pressurised to near full vacuum $(-30 \mathrm{mmHg})$. A flow controller (Restek Corporation, PA, USA) was used to maintain a flow rate of $0.9 \mathrm{ml} / \mathrm{min}$ and sampling was stopped when air pressure in the canisters reached between -5 and $-3 \mathrm{mmHg}$. Samples were analysed using real-time gas chromatography mass spectrometry (GCMS) for toluene, xylene, styrene, acetone, methyl and ethyl-acetates and benzene. The limit of detection was 5 ppm and samples below this limit were assigned a value of $2.5 \mathrm{ppm}$. Exposure measurements were analysed by aggregating the concentrations of the individual compounds detected. In addition, the additive limit value (ALV) was calculated by assigning a weight to each individual compound detected, based upon its relative workplace exposure standard (American Governmental Conference of Industrial Hygienists' Threshold Limit Values [23]), and summing these values together. Where the ALV was $>1$, it was deemed that the exposure standard for that combination of compounds had been exceeded [23]. 


\section{Statistical analyses}

All statistical analyses were conducted using Stata version 13.1 (StataCorp LP, Texas, USA). As exposure data were not normally distributed we used geometric mean concentrations and standard deviations to summarise exposures for each of the collision repair groups (Office staff, spray painters and panel beaters). Symptoms of neurotoxicity were initially grouped on the basis of the total number of positive symptoms reported i.e. $\geq 5$, $\geq 10$, or $\geq 15$. For symptoms clustered in specific domains (described above) we used a cut-point of $\geq 3$ positive symptoms, which is an approach previously shown to be highly sensitive and specific in the classification of CSN patients[21]

Prevalence ORs comparing symptoms between collision repair workers and the reference population were calculated using logistic regression. All analyses were adjusted for age, ethnicity, smoking, alcohol consumption, education status and personality trait score [21]. Other potential confounders including sleep quality, chronic diseases (e.g. diabetes), head injuries, concussion, chronic fatigue, prescription drug use and pre-existing health issues were also considered, but these did not appreciably affect the observed associations. Sensitivity analyses were conducted excluding reference workers who reported some exposure to solvents.

The effect of work duration was assessed by dividing collision repair workers into tertiles, but these were then 'rounded off' to the nearest units of work duration i.e. those who had worked in the industry for less than 10 years (an-average of 5.4 years; frange $0.3-10.4$ ), 10 -19 years (average 14.8 years $(10.4-21.0)$ ), and more than 20 years (average 31.3 years $(21.0-50.0)$ ). Due to the high correlation of age with employment duration (Spearman's correlation coefficient $=0.89)$ and the resulting potential for multicolinearity, two regression models were used i.e. one which controlled for all confounders except age (model 1), and one controlling for all confounders including age (model 2).

Commented [n5]: The multicollinearity seems to be very minor, and normally we would always want to adjust for age; could we just use model 2 throughout and note in the text that there were only differences between the results for model 1 and model 2 ? 


\section{RESULTS}

The response rates for the collision repair workers and the reference population were $69 \%$ and $64 \%$ respectively. Of 397 collision repair workers who agreed to participate 7 fulfilled the exclusion criteria and 3 of the 222 reference workers were excluded for previously working as spray painters. Also, 20 collision repair workers and 4 reference group workers were unable to complete the interview. Complete data was therefore available for 370 collision repair workers ( 234 spray painters, 90 panel beaters and 46 office workers who were previously employed in either role) and 215 reference workers. Fifty-three collision repair workers who declined to participate completed the non-respondent questionnaire and no appreciable differences in age, ethnicity, smoking habits, and key neurobehavioural symptoms were found between those who participated and those who declined (data not shown).

Collision repair workers included a higher proportion of Māori, were marginally older, smoked less, and consumed less alcohol than the reference group. Also, fewer had completed a tertiary degree, and they also scored higher on the personality trait scales (Table 1). All analyses were therefore controlled for these factors. Within the collision repair group, panel beaters were slightly older and had a higher proportion of Pacific people than spray painters (Table 1$)$

The majority of exposure measurements showed detectable levels of toluene, xylene, acetone and butyl acetate, with a smaller proportion also indicating the presence of hexane, methyl ethyl ketone, and methyl isobutyl ketone (data not shown). Overall, airborne solvent concentrations were low and below the American Conference of Governmental and Industrial Hygienists' TLVs (ACGIH, TLVs)[23]. Solvent levels were highest in spray painters (geometric mean combined solvent level of $2.26 \mathrm{ppm}$ ) followed by panel beaters $(0.57 \mathrm{ppm})$ and office staff (0.19 ppm; Table 2). The additive limit values for the solvent mixtures were also generally low (Table 2).

Collision repair workers reported significantly more symptoms of neurotoxicity than reference workers with ORs of 2.0, 2.4 and 6.4 (all $p<0.05$ ) for reporting $\geq 5, \geq 10$ and $\geq 15$ symptoms respectively (Table 3 ). Associations 
with specific symptom domains (using cut-points of $\geq 3$ positive symptoms) showed increased risks for neurological (OR 4.2), psychosomatic (OR 3.2), mood (OR 2.1), memory (OR 2.9) and memory and concentration symptoms combined (OR 2.4) (all p<0.05; Table 3). Generally, the strongest associations were observed in panel beaters. Highly comparable results were found when different cut-offs (e.g. $\geq 2$ or $\geq 4$ symptoms) were used (data not shown). When comparing workers based on employment duration, those with medium employment duration reported the greatest number of symptoms followed by those with the longest employment duration. Those with the shortest employment duration had the least number of symptoms, but they still reported more symptoms than the reference population (Table 4). Using alternative stratifications (i.e. using quartiles or quintiles) did not significantly alter the results (data not shown). Adjusting for age (model 2) altered the standard error for some outcomes, but the effect on risk estimates and trends was negligible (Table 4). We also repeated all analyses excluding current office workers (who previously worked as a spray painter/panel beater) and this also did not affect the results (data not shown).

Collision repair workers reported more acute symptoms including eye, mouth and throat dryness/irritation, feeling drunk without drinking and unpleasant taste in the mouth, but these did not reach statistical significance (Table 5). In contrast, collision repair workers were less likely to report sensitivity to environmental stimuli including strong smells, heat, and cold $(p<0.05)$ with most pronounced differences observed in spray painters (Table 5). 


\section{DISCUSSION}

Collision repair workers consistently reported more symptoms of neurotoxicity than reference workers, primarily in the domains of neurological, psychosomatic, mood and memory symptoms. Differences were most pronounced in panel beaters. The strongest risks were observed in workers with medium employment duration (15 years) in the collision repair industry compared to those with shorter ( 5 years) or longer ( 31 years) work duration.

The current study has shown an elevated risk of neurotoxicity in collision repair workers consistent with previous studies in car spray painters, particularly those that also focused on smaller enterprises [9 24],

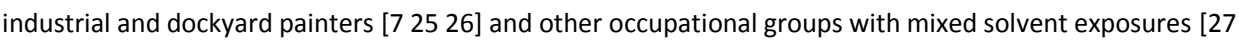
28]. The increased reports of neurological symptoms suggest peripheral neuropathy which has been shown to be associated with long-term exposure to solvents in case studies[29] and a cross-sectional study of industrial painters showing an increased risk of leg and arm paraesthesia (abnormal sensation, pins and needles) [7].

The increased risk of neurotoxicity observed in this study appears to occur at airborne solvent levels below international exposure standards. D, but due to the potential long latency of solvent-related neurotoxic effects a role for high historic exposures cannot be excluded. However, increased risks are also observed in those with a short employment duration (Table 4), suggesting that symptoms are not solely attributable to historic exposures. Also, as observed internationally, significant reductions in solvent exposure levels in this industry are likely to have occurred as early as two decades ago [19] suggesting that contemporary low-level solvent exposure may indeed have contributed to the observed elevated risks, particularly given that the strongest associations were found in those who worked on average 15 years in the collision repair industry (i.e. after the reported reduction in exposure levels seen in this industry). If true, effects may be due to short duration high peak exposures which contribute little to full-shift average exposures as measured in the current study, but may act as a "tipping point" in the development of neurotoxic symptoms [30]. Alternatively, airborne exposures do not accurately reflect the total solvent burden, and dermal exposures (which we have not measured) may be more important. Also, it is possible that, instead of, or in addition to chronic low-level 
solvent exposure, the frequent use of vibrating air-powered and oscillating tools, particularly by panel beaters, may have contributed to some of the observed increased risks [26 31]. However, this would only apply to neurological symptoms and not to other neurobehavioural symptoms, which were also more frequently reported by collision repair workers.

The strongest risks of neurotoxicity were observed in panel beaters despite no direct involvement in the painting process, and detected airborne levels being only a quarter of those in spray painters. It is unclear why this is the case, but it may be due to exposure to solvents not tested for. In particular, the panel repair process involves regular use of heavy duty cleaning and degreasing aerosol sprays which often contain chlorinated solvents such as perchloroethylene, tetrachloroethylene and methylene chloride, which have been associated with neurobehavioural and neurological effects[32]. Air samples were not tested for these chemicals. Panel beating was also often performed in close proximity to spray painting activities and in confined spaces, such as the interior of vehicles. Furthermore, panel beaters rarely wore suitable respirators (unlike spray painters). The differences may also be due to differences in historic exposures between spray painters and panel beaters [9], but this could not be assessed in this cross-sectional study. Finally, as noted above, dermal exposures may be more important than airborne exposures and this may be particularly the case for panel beaters.

Collision repair workers, and particularly spray painters, reported less sensitivity to environmental conditions (table 5) than the reference group, including sensitivity to strong smells. Deficits in olfactory function have previously been shown in solvent exposed workers, and effects on olfactory neuroepithelial function are suspected[33]. Reduced sensation to rough fabrics next to the skin, and heat and cold were also reported less by spray painters, which may be indicative of altered CNS function [1].

Those working in the collision and repair industry for a medium duration had a greater risk of reporting symptoms overall as well as for each of the symptom domains separately compared to those with short and long employment duration. Despite signs of multicolinearity (with confidence limits widening), this trend was unaffected when the analyses were adjusted for age, suggesting that employment duration is, at least to some 
degree, independently associated with symptoms. However, a clear dose-response was not observed which may be due to 'healthy worker survivor bias', a selection phenomenon, where those least susceptible to the effects of solvents continue work in high exposure jobs, whereas those who develop symptoms leave the industry or move to lower exposed jobs [15]. Evidence of this effect has been reported in several other studies of solvent exposed workers [34 35] including a 2008 meta-analysis[15]. Also, the lack of a clear exposure response trend is consistent with the conclusions from a review of studies looking at low-level hydrocarbon exposures and neurobehavioural effects[18].

The response rate in collision repair and reference workers was $69 \%$ and $64 \%$ respectively, which is relatively high for these types of surveys and suggests that non-response bias, if present, would be small. Also, no differences between responders and non-responders were found for several key symptoms of neurotoxicity further suggesting that non-response bias is negligible and unlikely to explain the increased risks observed in collision repair workers. However, this study had other limitations. In particular, it is possible that some reference workers were, or had previously been, occupationally exposed to solvents. If that was the case the risks observed in the collision repair group might be an underestimate of the true risks. However, analyses excluding reference workers who reported occupational exposure to solvents did not alter the results (data not shown), suggesting that this is not a major issue. As noted above, there was some evidence of a healthy worker survivor bias, but this is also likely to result in an underestimation of risk. There were differences in age, ethnicity, attained education, smoking habits, and alcohol consumption between collision repair workers and reference workers, but these were controlled for in the analyses, and study results were consistent with previous international studies suggesting that results are robust. Duration of employment in the industry is generally not considered the most reliable proxy for cumulative exposure[18], but historical data of solvent levels in this industry was not available and exposure misclassification would likely lead to an underestimation of risk. Finally neurotoxic effects were assessed using self-reported symptoms, which were not confirmed by a clinical assessment, and therefore some misclassification may have occurred. However, EUROQUEST was designed specifically to assess symptoms associated with occupational exposure to neurotoxic agents [36], and is widely used and well validated against clinical criteria [20 21 37-39]. Also, the same questionnaires were 
used in collision repair workers and the reference population and administered according to the same standardised protocol, and comparisons are therefore valid.

In conclusion, despite solvent exposures in the collision repair industry having declined steadily over the past two decades internationally and current airborne exposures in New Zealand being well under international standards, collision repair workers in small to medium-sized enterprises continue to have a significantly elevated risk of neurotoxicity. Thus, further preventive measures may need to be implemented to reduce hazardous exposures and associated neurotoxicity in the collision repair industry. 


\section{ACKNOWLEDGEMENTS}

We would like to thank the study participants and their employers and the New Zealand Collision Repair Association (CRA) for their generous support, and Leigh Emmerton, Angela Thurston, Tracey Whaanga, Hannah Buchanan, Emma Nuttall and Phoebe Taptiklis for conducting interviews and exposure measurements. The Centre for Public Health Research is funded by a Programme Grant from the Health Research Council (HRC) of New Zealand, which included this study. 


\section{REFERENCES}

1. Dick FD. Solvent neurotoxicity. Occupational and environmental medicine 2006;63(3):221-6, 179

2. Baker EL, Fine LJ. Solvent Neurotoxicity: The Current Evidence. Journal of Occupational and Environmental Medicine 1986;28(2):126-70

3. Chronic Organic Solvent Neurotoxicity: Diagnostic Criteria. Wellington, New Zealand: Occupational Safety and Health Service, New Zealand Department of Labour, 1992, 1998.

4. Dryson EW, Ogden JA. Chronic solvent neurotoxicity in New Zealand: notified cases between 1993 and 1997. New Zeal Med J 1998;111(1077):425-27

5. Keski-Santti P, Kaukiainen A, Hyvarinen HK, et al. Occupational chronic solvent encephalopathy in Finland 1995-2007: incidence and exposure. International archives of occupational and environmental health 2010;83(6):703-12

6. Hanninen H, Eskelinen L, Husman K, et al. Behavioral effects of long-term exposure to a mixture of organic solvents. Scandinavian journal of work, environment \& health 1976;2(4):240-55

7. Elofsson SA, Gamberale F, Hindmarsh T, et al. Exposure to organic solvents. A cross-sectional epidemiologic investigation on occupationally exposed care and industrial spray painters with special reference to the nervous system. Scandinavian journal of work, environment \& health 1980;6(4):239-73

8. Husman K. Symptoms of car painters with long-term exposure to a mixture of organic solvents. Scandinavian journal of work, environment \& health 1980;6(1):19-32

9. Daniell W, Stebbins A, O'Donnell J, et al. Neuropsychological performance and solvent exposure among car body repair shop workers. British journal of industrial medicine 1993;50(4):368-77

10. Hanninen $\mathrm{H}$, Antti-Poika M, Juntunen J, et al. Exposure to organic solvents and neuropsychological dysfunction: a study on monozygotic twins. British journal of industrial medicine 1991;48(1):18-25

11. Triebig G, Schaller KH, Weltle D. Neurotoxicity of solvent mixtures in spray painters. I. Study design, workplace exposure, and questionnaire. International archives of occupational and environmental health 1992;64(5):353-9

12. Triebig G, Barocka A, Erbguth F, et al. Neurotoxicity of solvent mixtures in spray painters. II. Neurologic, psychiatric, psychological, and neuroradiologic findings. International archives of occupational and environmental health 1992;64(5):361-72

13. Maizlish NA, Langolf GD, Whitehead LW, et al. Behavioural evaluation of workers exposed to mixtures of organic solvents. British journal of industrial medicine 1985;42(9):579-90

14. Chen R, Dick F, Semple S, et al. Exposure to organic solvents and personality. Occupational and environmental medicine 2001;58(1):14-8

15. Meyer-Baron $M$, Blaszkewicz $M$, Henke $H$, et al. The impact of solvent mixtures on neurobehavioral performance: conclusions from epidemiological data. Neurotoxicology 2008;29(3):349-60

16. Hasle P, Limborg HJ, oslash, et al. A Review of the Literature on Preventive Occupational Health and Safety Activities in Small Enterprises. Industrial health 2006;44(1):6-12 doi: 10.2486/indhealth.44.6[published Online First: Epub Date]|.

17. Mikkelsen S. Epidemiological update on solvent neurotoxicity. Environmental research 1997;73(1-2):10112

18. Gamble JF. Low-level hydrocarbon solvent exposure and neurobehavioural effects. Occupational medicine 2000;50(2):81-102

19. Kauppinen T, Uuksulainen S, Saalo A, et al. Trends of Occupational Exposure to Chemical Agents in Finland in 1950-2020. Ann Occup Hyg 2013;57(5):593-609

20. Carter N, Iregren A, Soderman E, et al. EUROQUEST--a questionnaire for solvent related symptoms: factor structure, item analysis and predictive validity. Neurotoxicology 2002;23(6):711-7

21. Kaukiainen A, Hyvarinen HK, Akila R, et al. Symptoms of chronic solvent encephalopathy: Euroquest questionnaire study. Neurotoxicology 2009;30(6):1187-94

22. Compendium Method TO-14A - Determination Of Volatile Organic Compounds (VOCs) In Ambient Air Using Specially Prepared Canisters With Subsequent Analysis By Gas Chromatography. Cinncinati, OH, USA.: Centre for Environmental Research Information, U.S. Environmental Protection Agency, 1999.

23. TLVs and BEIs: Threshold limit values for chemical substances and physical agents biological exposure indices. Cincinnati, OH.: American Conference of Governmental Industrial Hygienists, 2015. 
24. Böckelmann I, Darius S, McGauran N, et al. The psychological effects of exposure to mixed organic solvents on car painters. Disability and Rehabilitation 2002;24(9):455-61

25. Chen R, Dick F, Seaton A. Health effects of solvent exposure among dockyard painters: mortality and neuropsychological symptoms. Occupational and environmental medicine 1999;56(6):383-7

26. Kishi R, Harabuchi I, Katakura Y, et al. Neurobehavioral Effects of Chronic Occupational Exposure to Organic Solvents Among Japanese Industrial Painters. Environmental research 1993;62(2):303-13

27. Böckelmann I, Pfister EA, Peters B, et al. Psychological effects of occupational exposure to organic solvent mixtures on printers. Disability and Rehabilitation 2004;26(13):798-807

28. White RF, Robins TG, Proctor S, et al. Neuropsychological effects of exposure to naphtha among automotive workers. Occupational and environmental medicine 1994;51(2):102-12

29. Dick F, Semple S, Chen R, et al. Neurological deficits in solvent-exposed painters: a syndrome including impaired colour vision, cognitive defects, tremor and loss of vibration sensation. QJM 2000;93(10):655-61

30. Checkoway $\mathrm{H}$, Rice $\mathrm{CH}$. Time-weighted averages, peaks, and other indices of exposure in occupational epidemiology. American Journal of Industrial Medicine 1992;21(1):25-33

31. Jaycock MA, Levin L. Health hazards in a small auto body repair shop. Ann Occup Hyg 1984;28(1):19-29

32. Seppalainen AM, Antti-Poika M. Time course of electrophysiological findings for patients with solvent poisoning: A descriptive study. Scandinavian journal of work, environment \& health 1983;9(1):15-24

33. Gobba F. Olfactory toxicity: long-term effects of occupational exposures. International archives of occupational and environmental health 2006;79(4):322-31

34. Foo SC, Lwin S, Chia SE, et al. Chronic neurobehavioural effects in paint formulators exposed to solvents and noise. Ann Acad Med Singapore 1994;23(5):650-54

35. Escalona E, Yanes L, Feo O, et al. Neurobehavioral evaluation of venezuelan workers exposed to organic solvent mixtures. American Journal of Industrial Medicine 1995;27(1):15-27

36. Karlson B, Osterberg K, Orbaek P. Euroquest: the validity of a new symptom questionnaire. Neurotoxicology 2000;21(5):783-9

37. Williamson A. Using self-report measures in neurobehavioural toxicology: Can they be trusted? Neurotoxicology 2007;28(2):227-34

38. Rouch I, Wild P, Fontana J-M, et al. Evaluation of the French Version of EUROQUEST: A Questionnaire for Neurotoxic Symptoms. Neurotoxicology 2003;24(4-5):541-46

39. Kaukiainen A, Akila R, Martikainen R, et al. Symptom screening in detection of occupational solvent-related encephalopathy. International archives of occupational and environmental health 2009;82(3):343-55 
Table 1. Demographic and work characteristics of study participantsfor groups-studied$$
\text { Male }
$$

Ethnicity

Maori

Pacific
Other (incl. NZ European)

Smoking Status

Non-smoker

Ex-smoker

Current smoker

Education level

primary

secondary

trade cert.

Tertiary

Age

Alcohol (Mean drinks per week)

Duration of employment (Yrs)

EUROQUEST personality score

\begin{tabular}{cccccccc}
\multicolumn{2}{c}{ Reference workers $(\mathbf{n}=\mathbf{2 1 5})$} & \multicolumn{2}{c}{ All Collision repair $(\mathbf{n = 3 7 0})$} & \multicolumn{2}{c}{ Spray painters $(\mathbf{n}=\mathbf{2 6 0})$} & \multicolumn{2}{c}{ Panel beaters $(\mathbf{n}=\mathbf{1 1 0})$} \\
\hline $\mathbf{n}$ & $\mathbf{\%}$ & $\mathbf{n}$ & $\mathbf{\%}$ & $\mathbf{n}$ & $\mathbf{\%}$ & $\mathbf{n}$ & $\mathbf{\%}$ \\
\hline & & & & & & & \\
214 & 99.5 & 369 & 99.7 & 259 & 99.5 & 110 & 100.0 \\
1 & 0.5 & 1 & 0.3 & 1 & 0.5 & 0 & 0.0 \\
& & & & & & & \\
70 & 32.6 & 50 & 13.5 & 37 & 14.2 & 13 & 11.8 \\
24 & 11.2 & 30 & 8.1 & 15 & 5.8 & 15 & 13.6 \\
121 & 56.3 & 290 & 78.4 & 208 & 80.0 & 82 & 74.6 \\
& & & & & & & \\
88 & 40.9 & 151 & 40.8 & 115 & 44.2 & 49 & 44.6 \\
47 & 21.9 & 109 & 29.5 & 69 & 26.5 & 27 & 24.6 \\
80 & 37.2 & 110 & 29.7 & 76 & 29.2 & 34 & 30.9 \\
& & & & & & & \\
4 & 1.9 & 10 & 2.7 & 5 & 1.9 & 5 & 4.6 \\
144 & 68.8 & 262 & 70.6 & 187 & 71.9 & 77 & 67.3 \\
51 & 23.7 & 85 & 22.9 & 58 & 22.3 & 27 & 24.6 \\
16 & 7.4 & 14 & 3.7 & 10 & 3.9 & 4 & 3.6 \\
& & & & & & & \\
Mean & Range & Mean & Range & Mean & Range & Mean & Range \\
36.3 & $17-66$ & 36.7 & $17-64$ & 35.8 & $17-64$ & 38.8 & $20-63$ \\
15.7 & $0-120$ & 13.5 & $0-140$ & 13.6 & $0-140$ & 13.7 & $0-106$ \\
- & - & 17.0 & $0.3-50$ & 16.4 & $0.3-50$ & 18.58 & $1-47.4$ \\
0.69 & $(0-5)$ & 0.79 & $(0-6)$ & 0.80 & $(0-6)$ & 0.76 & $(0-6)$ \\
\hline
\end{tabular}

Commented [n6]: We should have a uniform order, e.g. always have panel beaters first and spray painters second (since they are supposed to have the higher exposures) 
Table 2. Full shift whole-air concentrations of all solvents detected combined (Geometric means), including Additive Limit Value (ALV) calculation.

Total Hydrocarbons (ppm)

Geometric Mean (Geometric SD)

Range

ALV calculated from ACGIH TLV's

Mean (SD)

Range
Office workers ( $n=6$ )

Mean (SD)

$0.19(2.1)$

$0.1-0.6$

Mean (SD)

$0.1-2.5$

$0.06(0.03)$

$0.04(0.01)$

$0.03-0.06$
Spray Painters $(\mathbf{n}=\mathbf{5 0})$

Mean (SD)

2.26 (2.6)

$0.1-16.6$

$0.15(0.12)$

$0.03-0.70$ 
Table 3. Prevalence odds ratios of dichotomised (yes/no) EUROQUEST symptoms between reference workers and all collision repair workers, and between reference workers and spray painters and panel beaters separately.

\begin{tabular}{|c|c|c|c|c|c|c|c|}
\hline \multirow[b]{2}{*}{ Cut-points } & \multirow{2}{*}{$\begin{array}{c}\text { Reference }(n=215) \\
n(\%)\end{array}$} & \multicolumn{2}{|c|}{ All CR workers ( $N=370$ ) } & \multicolumn{2}{|c|}{ Spray Painters $(n=267)$} & \multicolumn{2}{|c|}{ Panel Beaters ( $N=103$ ) } \\
\hline & & $n(\%)$ & $\mathrm{OR}^{*}(95 \% \mathrm{Cl})$ & $n(\%)$ & $\mathrm{OR}^{*}(95 \% \mathrm{Cl})$ & $n(\%)$ & OR* $(95 \% \mathrm{Cl})$ \\
\hline$\geq 3$ symptoms & $79(36.7)$ & $184(49.7)$ & $1.6(1.1-2.3)$ & $133(49.8)$ & $1.5(1.0-2.3)$ & $51(49.5)$ & $1.6(0.9-2.8)$ \\
\hline$\geq 5$ symptoms & 49 (22.8) & $140(37.8)$ & $2.0(1.3-3.3)$ & 99 (37.1) & $1.9(1.2-3.1)$ & $41(39.8)$ & $2.1(1.2-3.8)$ \\
\hline$\geq 10$ symptoms & $14(6.5)$ & 54 (14.6) & $2.4(1.2-4.8)$ & 35 (13.1) & $2.1(1.0-4.6)$ & $19(18.5)$ & $3.3(1.4-7.8)$ \\
\hline$\geq 15$ symptoms & $4(1.9)$ & $28(7.6)$ & $6.4(1.8-23.0)$ & $17(6.4)$ & $6.3(1.5-27.4)$ & $11(10.6)$ & $8.2(2.2-31.0)$ \\
\hline \multicolumn{8}{|l|}{ Symptom domains } \\
\hline$\geq 3$ Neurological & $3(1.4)$ & $19(5.1)$ & $4.2(1.2-15.3)$ & $11(4.1)$ & $3.2(0.8-13.2)$ & $8(7.8)$ & $5.4(1.3-22.6)$ \\
\hline$\geq 3$ Psychosomatic & $6(2.8)$ & $27(7.3)$ & $3.2(1.2-9.1)$ & $17(6.4)$ & $2.5(0.8-7.6)$ & $10(9.7)$ & $4.1(1.3-13.2)$ \\
\hline$\geq 3 \mathrm{Mood}$ & $12(5.6)$ & 45 (12.2) & $2.1(1.0-4.3)$ & 31 (11.6) & $1.9(0.9-4.2)$ & $14(13.6)$ & $2.7(1.1-6.7)$ \\
\hline$\geq 3$ Memory & $7(3.3)$ & $37(10.0)$ & $2.9(1.2-7.0)$ & $21(7.9)$ & $1.9(0.9-4.0)$ & $16(15.5)$ & $5.9(2.1-16.8)$ \\
\hline$\geq 3$ Concentration & $1(0.47)$ & $5(1.4)$ & $3.3(0.2-48.7)$ & $4(1.5)$ & $2.1(0.8-5.9)$ & $1(1.0)$ & $1.7(0.1-50.3)$ \\
\hline$\geq 3$ Fatigue & $20(9.3)$ & 48 (12.9) & $1.4(0.7-2.5)$ & 35 (13.1) & $1.4(0.7-2.8)$ & $13(12.6)$ & $1.2(0.5-2.8)$ \\
\hline$\geq 3$ Sleep Disturbance & $12(5.6)$ & $35(9.5)$ & $1.8(0.9-3.7)$ & $26(9.7)$ & $2.0(0.9-4.4)$ & $9(8.7)$ & $1.7(0.7-4.3)$ \\
\hline$\geq 3$ Memory and Conc. & $13(6.1)$ & 54 (14.6) & $2.4(1.2-4.8)$ & 34 (12.7) & $3.9(0.3-59.2)$ & 20 (19.4) & $4.3(1.8-10.3)$ \\
\hline
\end{tabular}

Odds ratios and confidence intervals in bold $-p=<0.05$

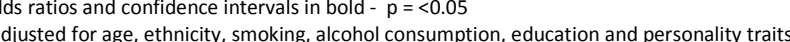


Table 4. Prevalence odds ratios of dichotomised (yes/no) EUROQUEST symptoms in reference workers and collision repair workers stratified by employment duration (tertiles) - Proportion of participants answering "Yes" to more than (x) number of symptoms, and more than 3 symptoms in each domain.

\begin{tabular}{|c|c|c|c|c|c|c|c|c|c|c|}
\hline \multirow[b]{3}{*}{ Cut-points } & \multirow{3}{*}{$\begin{array}{c}\begin{array}{c}\text { Reference group } \\
\mathrm{n}=215\end{array} \\
\mathrm{n}(\%) \\
\end{array}$} & \multicolumn{3}{|c|}{$\begin{array}{l}\text { Mean employment duration } 5.35 \text { (SD 2.91) } \\
n=125\end{array}$} & \multicolumn{3}{|c|}{$\begin{array}{c}\text { Mean employment duration } 14.75 \text { (SD 3.12) } \\
\mathrm{n}=123\end{array}$} & \multicolumn{3}{|c|}{$\begin{array}{c}\text { Mean employment duration } 31.3 \text { (SD 7.29) } \\
\mathrm{n}=122\end{array}$} \\
\hline & & \multirow[t]{2}{*}{$n(\%)$} & \multicolumn{2}{|c|}{ OR (95\% Cl) } & \multirow[t]{2}{*}{$n(\%)$} & \multicolumn{2}{|c|}{ OR $(95 \% \mathrm{Cl})$} & \multirow[t]{2}{*}{$n(\%)$} & \multicolumn{2}{|c|}{ OR $(95 \% \mathrm{Cl})$} \\
\hline & & & Model 1* & Model $2^{+}$ & & Model 1* & Model $2^{+}$ & & Model 1* & Model $2^{+}$ \\
\hline$\geq 3$ symptoms & $79(36.7)$ & $54(43.2)$ & $1.1(0.7-1.9)$ & $1.0(0.6-1.7)$ & $70(57.4)$ & $2.3(1.4-3.8)$ & $2.2(1.3-3.7)$ & $60(48.4)$ & $1.5(0.9-2.5)$ & $1.7(1.0-3.0)$ \\
\hline$\geq 5$ symptoms & 49 (22.8) & $38(30.4)$ & $1.3(0.8-2.3)$ & $1.3(0.7-2.3)$ & $58(47.5)$ & $3.2(1.9-5.5)$ & $3.2(1.9-5.6)$ & 44 (35.5) & $1.7(1.0-3.0)$ & $1.9(1.0-3.4)$ \\
\hline$\geq 10$ symptoms & $14(6.5)$ & $15(12.0)$ & $1.9(0.8-4.3)$ & $1.7(0.7-4.2)$ & $22(18.0)$ & $3.4(1.5-7.6)$ & $3.3(1.5-7.5)$ & 17 (13.7) & $2.3(1.0-5.4)$ & $2.5(0.9-6.3)$ \\
\hline$\geq 15$ symptoms & $4(1.9)$ & $5(4)$ & $2.4(0.5-11.5)$ & $1.9(0.3-10.1)$ & 14 (11.5) & $12.2(3.1-47.4)$ & $11.5(3.0-44.6)$ & $9(7.3)$ & $6.2(1.4-26.5)$ & $7.6(1.5-37.9)$ \\
\hline \multicolumn{11}{|l|}{ Symptom Domains } \\
\hline$\geq 3$ Neurological & $3(1.4)$ & $3(2.4)$ & $1.7(0.3-8.9)$ & $1.8(0.3-11.4)$ & $9(7.4)$ & $6.1(1.5-24.4)$ & $6.2(1.5-25.4)$ & $7(5.7)$ & $5.0(1.1-21.5)$ & $4.5(0.9-22.4)$ \\
\hline$\geq 3$ Psychosomatic & $6(2.8)$ & $4(3.2)$ & $1.2(0.3-4.8)$ & $1.0(0.2-4.4)$ & $12(9.8)$ & $5.2(1.7-16.4)$ & $5.0(1.6-15.8)$ & $11(8.9)$ & $4.0(1.2-13.2)$ & $4.7(1.2-18.0)$ \\
\hline$\geq 3$ Mood & $12(5.6)$ & $11(8.8)$ & $1.6(0.6-3.9)$ & $1.1(0.4-2.9)$ & $23(18.9)$ & $3.9(1.7-8.7)$ & $3.7(1.6-8.3)$ & $11(8.9)$ & $1.4(0.5-3.6)$ & $2.2(0.7-6.5)$ \\
\hline$\geq 3$ Memory & $1(0.47)$ & $11(8.8)$ & $2.6(0.9-7.6)$ & $1.7(0.6-5.2)$ & $13(10.7)$ & $3.6(1.3-10.1)$ & $3.3(1.2-9.3)$ & $13(10.5)$ & $2.8(1.0-8.2)$ & $4.9(1.4-17.5)$ \\
\hline$\geq 3$ Concentration & $13(6.1)$ & $2(1.6)$ & $5.4(0.3-104.7)$ & $0.6(0-16)$ & $2(1.6)$ & $3.8(0.2-66.7)$ & - & $1(0.81)$ & $2.3(0.1-63.9)$ & - \\
\hline$\geq 3$ Fatigue & $20(9.3)$ & $8(6.4)$ & $0.6(0.2-1.4)$ & $0.5(0.2-1.4)$ & $20(16.4)$ & $1.9(0.9-4.1)$ & $1.9(0.9-4.0)$ & $20(16.1)$ & $1.8(0.8-4.0)$ & $2.0(0.8-4.6)$ \\
\hline$\geq 3$ Sleep Disturbance & $12(5.6)$ & $6(4.8)$ & $0.8(0.3-2.3)$ & $1.1(0.3-3.2)$ & $12(9.8)$ & $1.8(0.8-4.4)$ & $2.0(0.8-4.9)$ & 17 (13.7) & $2.8(1.2-6.6)$ & $2.2(0.9-5.5)$ \\
\hline$\geq 3$ Memory and Conc. & $7(3.3)$ & $17(13.6)$ & $2.4(1.1-5.5)$ & $1.5(0.6-3.6)$ & $23(18.9)$ & $3.6(1.6-8.0)$ & $3.5(1.5-7.8)$ & $14(11.3)$ & $1.6(0.7-3.9)$ & $3.0(1.0-8.9)$ \\
\hline
\end{tabular}

Odds Ratios in bold are statistically significant $(p<0.05)$

* Model 1 adjusted for ethnicity, smoking, alcohol consumption, education \& personality traits 
Table 5. Prevalence odds ratios of dichotomised (yes/no) acute symptom and sensitivity to environmental conditions EUROQUEST questions between reference workers and all collision repair workers, and between reference workers and spray painters and panel beater $s$ separately.

\begin{tabular}{|c|c|c|c|c|c|c|c|}
\hline & \multirow{2}{*}{$\begin{array}{c}\text { Reference } \mathrm{n}=\mathbf{2 1 5} \\
\mathrm{n}(\%)\end{array}$} & \multicolumn{2}{|c|}{ All CR $\mathrm{n}=\mathbf{3 7 0}$} & \multicolumn{2}{|c|}{ Spray Painters $n=260$} & \multicolumn{2}{|c|}{ Panel Beaters $n=110$} \\
\hline & & $n(\%)$ & OR*(95\% Cl) & $n(\%)$ & $\mathrm{OR}^{*}(95 \% \mathrm{Cl})$ & $n(\%)$ & OR*(95\% Cl) \\
\hline \multicolumn{8}{|l|}{ Acute EQ symptoms } \\
\hline 1. Irritation of eyes & $18(8.5)$ & 39 (10.6) & $1.2(0.7-2.2)$ & $28(10.8)$ & $1.2(0.6-2.4)$ & $11(10.0)$ & $1.1(0.5-2.5)$ \\
\hline 2. Feeling drunk w/o. drinking & $0(0.0)$ & $3(0.8)$ & - & $1(0.4)$ & - & $2(1.8)$ & - \\
\hline 3. Dryness mouth/throat & $18(8.4)$ & $41(11.1)$ & $1.4(0.7-2.6)$ & $32(12.3)$ & $1.4(0.7-2.8)$ & $9(8.2)$ & $1.1(0.5-2.8)$ \\
\hline 4. Throat irritation & $8(3.7)$ & $26(7.0)$ & $1.7(0.7-4.0)$ & $16(6.2)$ & $1.4(0.6-3.5)$ & $10(9.1)$ & $2.5(0.9-7.3)$ \\
\hline 5. A runny nose & $21(9.8)$ & $24(6.5)$ & $0.6(0.3-1.1)$ & $11(4.3)$ & $0.5(0.2-1.0)$ & $13(11.8)$ & $0.9(0.4-2.2)$ \\
\hline 6: Unpleasant taste in mouth & $4(1.9)$ & $13(3.5)$ & $1.9(0.6-6.7)$ & $8(3.1)$ & $1.6(0.4-6.3)$ & $5(4.6)$ & $2.8(0.5-14.0)$ \\
\hline \multicolumn{8}{|c|}{ Sensitivity to environmental conditions } \\
\hline 1. Bright lights & 69 (32.4) & $123(33.2)$ & $1.1(0.8-1.6)$ & $78(30.0)$ & $0.99(0.7-1.5)$ & 45 (40.9) & $1.5(0.9-2.5)$ \\
\hline 2. Traffic noise, loud music, etc. & $53(24.9)$ & $77(20.8)$ & $0.7(0.5-1.1)$ & $51(19.6)$ & $0.71(0.4-1.2)$ & $26(23.6)$ & $0.8(0.5-1.5)$ \\
\hline 3. strong smells & $84(39.6)$ & $108(29.2)$ & $0.6(0.4-0.9)$ & $69(26.5)$ & $0.6(0.4-0.9)$ & $39(35.5)$ & $0.7(0.4-1.2)$ \\
\hline 4. Rough fabrics next to skin & $65(30.5)$ & $85(23.1)$ & $0.7(0.5-1.0)$ & $53(20.5)$ & $0.6(0.4-1.0)$ & $32(29.4)$ & $0.8(0.5-1.5)$ \\
\hline 5. Heat & $83(39.0)$ & $78(21.1)$ & $0.4(0.3-0.6)$ & 48 (18.5) & $0.3(0.2-0.5)$ & $30(27.3)$ & $0.5(0.3-0.9)$ \\
\hline 6. Cold & $81(38.0)$ & $91(24.6)$ & $0.5(0.3-0.7)$ & $54(20.8)$ & $0.4(0.2-0.6)$ & $37(33.6)$ & $0.6(0.4-1.1)$ \\
\hline
\end{tabular}

Odds Ratios in bold are statistically significant $(\mathrm{p}<0.05)$

"Adjusted for age, ethnicity, smoking, alcohol consumption, education \& personality traits 